\title{
Tractography of developing white matter of the internal capsule and corpus callosum in very preterm infants
}

\author{
Francisca T. de Bruïne • Gerda van Wezel-Meijler • Lara M. Leijser • \\ Annette A. van den Berg-Huysmans • Andrea van Steenis • Mark A. van Buchem • \\ Jeroen van der Grond
}

Received: 17 March 2010 /Revised: 7 June 2010 / Accepted: 28 July 2010 / Published online: 12 September 2010

(C) The Author(s) 2010. This article is published with open access at Springerlink.com

\begin{abstract}
Objectives To investigate in preterm infants associations between Diffusion Tensor Imaging (DTI) parameters of the posterior limb of the internal capsule (PLIC) and corpus callosum (CC) and age, white matter (WM) injury and clinical factors.

Methods In 84 preterm infants DTI was performed between 40-62 weeks postmenstrual age on 3 T MR. Fractional anisotropy (FA), apparent diffusion coefficient (ADC) values and fibre lengths through the PLIC and the genu and splenium were determined. WM injury was categorised as normal/mildly, moderately and severely abnormal. Associations between DTI parameters and age, WM injury and clinical factors were analysed.

Results A positive association existed between FA and age at imaging for fibres through the PLIC $(r=0.48 p<0.001)$ and splenium $(r=0.24 p<0.01)$. A negative association existed between ADC and age at imaging for fibres through the PLIC $(r=-0.65 p<0.001)$, splenium $(r=-0.35 p<0.001)$ and genu $(r=-0.53 p<0.001)$. No association was found between DTI parameters and gestational age, degree of WM injury or categorical clinical factors.

Conclusions These results indicate that in our cohort of very preterm infants, at this young age, the development of the PLIC and CC is ongoing and independent of the degree of prematurity or WM injury.
\end{abstract}

F. T. de Bruïne $(\bowtie) \cdot$ A. A. van den Berg-Huysmans

M. A. van Buchem · J. van der Grond

Department of Radiology, Leiden University Medical Center, PO Box 9600, 2300 RC Leiden, The Netherlands

e-mail: F.T.Wiggers-de_Bruine@lumc.nl

G. van Wezel-Meijler $\cdot$ L. M. Leijser · A. van Steenis Department of Neonatology, Leiden University Medical Center, Leiden, The Netherlands
Keywords Diffusion tensor imaging · Fibre tractography · Very preterm infants $\cdot$ Term equivalent age $\cdot$ White matter injury

$\begin{array}{ll}\text { Abbreviations } \\ \text { ADC } & \text { apparent diffusion coefficient } \\ \text { BPD } & \text { bronchopulmonary dysplasia } \\ \text { CC } & \text { corpus callosum } \\ \text { DEHSI } & \text { diffuse and excessive high signal intensity } \\ \text { DWI } & \text { diffusion-weighted imaging } \\ \text { DTI } & \text { diffusion tensor imaging } \\ \text { FA } & \text { fractional anisotropy } \\ \text { GA } & \text { gestational age } \\ \text { HC } & \text { head circumference } \\ \text { IUGR } & \text { intrauterine growth restriction } \\ \text { PDA } & \text { persistent ductus arteriosus } \\ \text { PLIC } & \text { posterior limb of internal capsule } \\ \text { PMA } & \text { postmenstrual age } \\ \text { PWML } & \text { punctuate white matter lesion } \\ \text { RDS } & \text { respiratory distress syndrome } \\ \text { TEA } & \text { term equivalent age } \\ \text { VPTI } & \text { very preterm infants } \\ \text { WM } & \text { white matter }\end{array}$

\section{Introduction}

Infants born at a gestational age (GA) below 32 weeks are prone to diffuse white matter (WM) injury $[1,2]$. This may eventually result in damage, underdevelopment and atrophy of the internal capsule and the corpus callosum (CC) [3-7]. Conventional MRI techniques detect WM injury and depict features of brain maturation [2, 8-15]. Diffusion Tensor Imaging (DTI) has been proposed as an additional tool in the assessment of WM injury and may provide more 
adequate diagnostic and prognostic information in relation to neurological outcome than conventional MR imaging [16-21]. DTI enables quantitative assessment of maturation, tract organisation and injury by calculating Fractional Anisotropy (FA) and Apparent Diffusion Coefficient (ADC) values. Fibre tractography offers insight into developing WM by visualisation of the WM tracts [1719, 22-25]. The non-invasive nature of DTI permits longitudinal and diagnostic clinical studies [26-33].

Until now, the clinical use of DTI fibre tractography in very preterm infants (VPTI) has been restricted by the lack of normal reference values for FA and ADC [16]. Only a few studies have used DTI in VPTI with a GA below 32 weeks [16, 18, 23-25]. In most of these studies MRI was performed before term equivalent age (TEA). Little is known about the changes in FA or ADC in the developing brain around TEA [18, 34-37]. Data on the influence of diffuse WM injury on tract maturation and organisation around TEA are scarce [35, 38, 39].

We studied DTI parameters (FA and ADC values and lengths of fibres) of WM tracts passing through the posterior limb of the internal capsule (PLIC) and the CC in VPTI undergoing MRI at or within the first months of TEA. The aims of this exploratory study were to establish the association between DTI parameters and:

- GA and postmenstrual age (PMA) at the time of imaging

- The degree of WM injury

- Categorical clinical factors that may influence brain maturation

\section{Materials and methods}

\section{Preterm infants}

As part of an ongoing prospective study of VPTI with a $\mathrm{GA} \leq 32$ weeks, admitted to the neonatal intensive care unit of our institution, 113 infants underwent MRI (3 Tesla) between May 2006 and October 2007.

MRI was preferably performed at TEA (PMA 4044 weeks). For infants who were unstable and/or ventilator dependent around that age, MRI was postponed (PMA range 40-62 weeks). Ethical approval for the study was given by the institutional review board and informed parental consent was obtained for each infant. In 102 infants DTI was performed. Three children with congenital brain abnormalities were excluded. In 15 cases, fibre tractography was not possible due to motion artefacts. In the remaining 84 infants a complete DTI dataset was acquired. Clinical parameters were retrospectively collected from the patient's medical records (Table 1).
Image and data acquisition

All MRI examinations were performed on a 3 Tesla MR system (Philips Medical Systems, Best, the Netherlands) according to a standard protocol [40]. The infants were sedated using chloral hydrate $(55 \mathrm{mg} / \mathrm{kg})$, laid supine and snugly swaddled during the imaging procedure. Ear protection consisted of neonatal earmuffs (Natus Mini Muffs; Natus Medical Inc., San Carlos, CA, USA) covered by a headphone. All MRI examinations included a 3D T1TFE sequence (TR $9.7 \mathrm{~ms}$, TE $4.6 \mathrm{~ms}$, flip angle 8, TFE factor 128, slice thickness $1 \mathrm{~mm}$ ) and a T2-TSE sequence (TR $6269 \mathrm{~ms}$, TE $120 \mathrm{~ms}$, turbo factor 18, slice thickness $2 \mathrm{~mm}$ ). In addition a DTI sequence (SE-EPI, TR 7,456 ms, TE $54 \mathrm{~ms}$, slice thickness $2 \mathrm{~mm}$, voxel size $1,4 \times 1,4 \times 2 \mathrm{~mm}$ with diffusion acquisitions in 32 directions and a $b$-value of $1,000 \mathrm{~s} / \mathrm{mm} 2$, EPI factor 56) with an imaging time of $5 \mathrm{~min}$ and $34 \mathrm{~s}$ was acquired.

Fibre tractography of the PLIC and CC

DTI datasets were analysed on an off-line workstation using commercially available processing software as provided by the manufacturer (FiberTrak, by Philips Medical Systems, Best, the Netherlands). DTI colour-coded maps were automatically computed, red representing a right-left, green an anterior-posterior and blue a superior-inferior orientation. These colour-coded DTI maps were used to place a single seed to perform fibre tracking using an automated 3D seeded algorithm. First, for the PLIC in the axial plane at the level of the lateral ventricles and subsequently for the genu and splenium of the $\mathrm{CC}$ in the mid-sagittal plane regions of interest were defined. The seed was placed as is shown in Fig. 1. Position changes of the seed-point in the structure of interest resulted in identical fibre tracts, indicating the robustness of the method used. Two investigators (FTdB and AvS, blinded to subject's age and degree of WM injury) identified the regions of interest and manually placed the individual seeds in consensus for each region of interest. Subsequently, fibre tracts were generated through the PLIC and the callosal genu and splenium resulting in visualisation of fibres (Fig. 2). The quality of all tracts was visually assessed in order to minimise erroneous pathways, which were erased and not used in further analyses. In all analyses, default settings were used consisting of a minimum FA of 0.15 , a maximum angle change of 27.0 degrees and a minimum fibre length of $10.0 \mathrm{~mm}$. We used the manufacturer default settings, since (small) changes of these settings, to optimize the performance of the fibre tracking, did not have any influence on the fibres picked by the tracking routine. Finally FA and ADC values of these fibre bundles were obtained and the length of the fibres was calculated. 
Table 1 Distribution of categorical clinical factors, white matter injury and continuous clinical parameters in the study population $(n=84)$

\begin{tabular}{lll}
\hline Categorical clinical factors & Number (N) & Percentage (\%) \\
\hline Male & 55 & 65.5 \\
Female & 29 & 34.5 \\
Plurality & 23 & 27.4 \\
IUGR & 10 & 11.9 \\
Antenatal corticosteroids & 43 & 51.2 \\
Perinatal infection & 30 & 35.7 \\
RDS & 44 & 52.4 \\
PDA & 23 & 27.4 \\
Hypotension & 29 & 34.5 \\
BPD & 41 & 48.8 \\
Use of dexamethasone for BPD & 11 & 13.1 \\
Degree of WM injury & & \\
Normal/mildly abnormal WM & 18 & 21.4 \\
Moderately abnormal WM & 51 & 60.7 \\
Severely abnormal WM & 15 & 17.8 \\
Continuous clinical parameters & Mean (SD) & Range \\
GA (weeks) & $29.0(2.0)$ & $25.6-31.9$ \\
Birth weight (g) & $1199(365)$ & $585-1960$ \\
Head circumference at birth (cm) & $25.8(4.0)$ & $22.0-31.2$ \\
PMA at MRI (weeks) & $45.0(4.2)$ & $40.0-62.1$ \\
Weight at MRI (gram) & $4002(867)$ & $2010-7005$ \\
Head circumference at MRI (cm) & $37.7(1.8)$ & $32.7-41.5$ \\
& & \\
\hline
\end{tabular}

$I U G R$ intrauterine growth restriction, $R D S$ respiratory distress syndrome, $P D A$ patent ductus arteriosus, $B P D$ bronchopulmonary dysplasia, $W M$ white matter, $G A$ gestational age, $P M A$ postmenstrual age

\section{WM injury}

All T1-W and T2-W sequences were analysed by two investigators (FTdB, paediatric neuroradiologist with more than 15 years of experience, LML, with more than 4 years' experience) by consensus to assess WM injury. We categorised the WM injury into [41]:

- Normal/mildly abnormal WM: normal appearing WM or homogeneous diffuse and excessive high signal intensity (DEHSI) as seen on T2-weighted images, or few $(\leq 6)$ punctate white matter lesions (PWML)

- Moderately abnormal WM: multiple (>6) PWML and/ or small localised cystic lesions and/or heterogeneous DEHSI

- Severely abnormal WM: extensive or diffuse heterogeneous signal intensity changes and/or haemorrhagic or cystic lesions involving the periventricular and/or subcortical WM

Accordingly, 18 infants were classified as having normal/mildly, 51 infants moderately and 15 infants severely abnormal WM (Table 1)
Statistical analysis

Data were analysed using SPSS 16.0.2 for Windows. Frequency counts and percentages were used to summarise categorical variables. For continuous variables, the mean, standard deviation of the mean and range are reported (Table 1).

Differences between DTI values of left and right PLIC and between the genu and splenium of the $\mathrm{CC}$ were determined by using Wilcoxon signed rank tests for related samples.

To establish the association between DTI parameters and age, weight and head circumference (HC) Spearman Rank correlation coefficients were calculated.

Stepwise linear regression analysis was used to assess the relative importance of the various study population characteristics that were associated with the DTI parameters through the PLIC and CC. Applying this model we corrected all other parameters for PMA at MRI and HC. Variables, for which the $p$ value by univariate analyses tests was $\leq 0.1$, were included in the subsequent linear stepwise regression analysis.

To determine the association between DTI parameters and WM injury and categorical clinical factors, Mann-Whitney U analyses or Kruskal-Wallis tests were performed where appropriate. One-way ANOVA with post-hoc Scheffe tests were used to compare DTI parameters between groups categorised as normal/mildly, moderately or severely abnormal WM.

A $p$ value $\leq 0.05$ was considered statistically significant for all analyses.

\section{Results}

General characteristics of the study population

Clinical characteristics for all 84 infants are shown in Table 1.

Quantitative DTI values

The FA and ADC values and length of fibres (mean, SD and range) through the PLIC and genu and splenium of the $\mathrm{CC}$ are shown in Table 2. As we found no statistical differences or any laterality between left and right DTI values in the PLIC, these DTI values were averaged. In the genu lower FA $(p<0.001)$, lower ADC values $(p<0.001)$ and shorter fibres $(p<0.001)$ were found compared with the splenium of the CC.

Association between DTI parameters and continuous clinical variables

Table 3 shows the associations between DTI parameters for the PLIC and genu and splenium and continuous clinical 
Fig. 1 a-b: a Axial colourcoded DTI map with right $(\rightarrow)$ and left $(\leftarrow)$ PLIC in blue and $\mathbf{b}$ sagittal colour-coded DTI map with $\mathrm{CC}$ in red, the regions of interest of genu $(\rightarrow)$ and splenium $(\leftarrow)$ are defined. The " $x$ " marks the place where a single seed point was placed in order to generate the fibre tracts

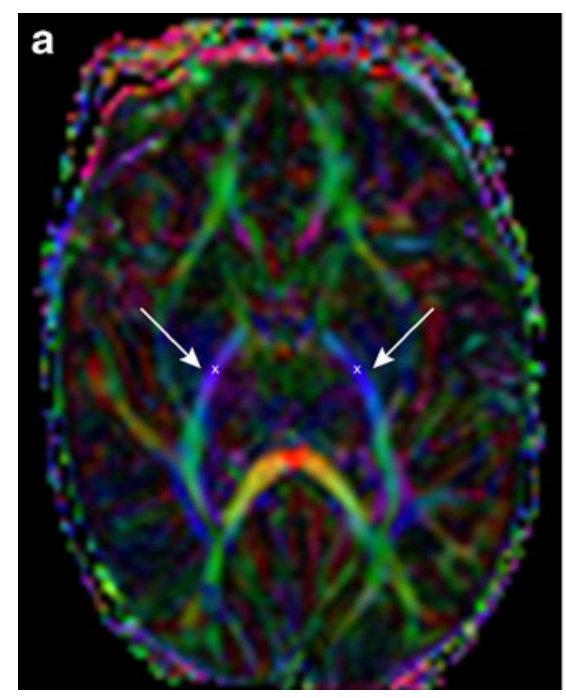

variables. DTI parameters in the PLIC $(p<0.001)$ and CC $(p<0.001$ and $p<0.01)$ correlated strongly with PMA at MRI, except for the FA in the genu of the CC. The FA ( $p<$ $0.01)$, ADC $(p<0.001)$ and fibre length $(p<0.01)$ through the PLIC and fibre length through the genu and splenium of the CC $(p<0.001, p<0.01$ and $p<0.001, p<0.001$ respectively) correlated with weight and HC at MRI. No or only weak associations were found between FA, ADC and fibre length through the PLIC or CC and GA or birth weight.

Because of the wide age range, we re-analysed data for a subgroup of infants (69/84) who underwent imaging between 40-46 weeks PMA. For this subgroup, we still found a significant association between FA $(r=0.252$ $p<0.05)$, ADC $(r=-0.470 p<0.01)$ and fibre length in the PLIC $(r=0.410 p<0.01)$ and PMA at MRI. The association between FA, ADC values or fibre length in the PLIC and weight and $\mathrm{HC}$ at MRI no longer existed. For the genu, no association between FA and any continuous clinical variable was found, while the association between ADC $(r=-0.268 p<0.05)$ or fibre length $(r=0.402 p<0.01)$ and PMA at MRI remained. For the splenium we found an association between fibre length and weight and $\mathrm{HC}$ at MRI (respectively $r=0.337 p<0.01$ and $r=0.332 p<0.05$ ). The association with PMA at MRI was no longer present, still the association between fibre length and $\mathrm{HC}$ at birth remained $(r=0.417 p<0.05)$.

Figure 3 shows the individual data depicting the association between the FA and ADC of fibres through the PLIC and GA as well as PMA at MRI. This figure illustrates the association between FA and ADC values with PMA at imaging, whereas no such association exists with GA.

Results of the stepwise regression analyses are shown in Table 4, delineating the strongest contributing factor of clinical parameters. In all but three cases, PMA at MRI was the strongest contributing factor to most of the DTI parameters (FA and ADC of the fibres through the PLIC, $\mathrm{ADC}$ and fibre length of the fibres through the genu and splenium, and FA of the splenium). For two of the remaining three parameters (FA in the fibres through the genu and fibre length in the fibres through the splenium) $\mathrm{HC}$ at birth contributed most. For the length of fibres through the PLIC, the HC at MRI contributed most.

Association between DTI parameters and WM injury and categorical clinical factors

We found no association between DTI parameters for the PLIC or CC and degree of WM injury. When comparing the groups with different degrees of WM injury, we also found no significant differences in DTI parameters (Table 5). These results remained, even after correction for PMA at MRI.

No associations existed between DTI parameters and categorical clinical factors.

\section{Discussion}

In this study, we determined FA and ADC values and the length of WM fibres passing through the PLIC and CC in a cohort of 84 VPTI imaged between 40 and 62 weeks PMA. We assessed the association between DTI parameters and age, diffuse WM injury and clinical factors.

All DTI parameters of the PLIC and most DTI parameters of the $\mathrm{CC}$ were strongly associated with PMA at imaging. We found no associations between DTI parameters and GA, the degree of WM injury or any categorical clinical factor. 


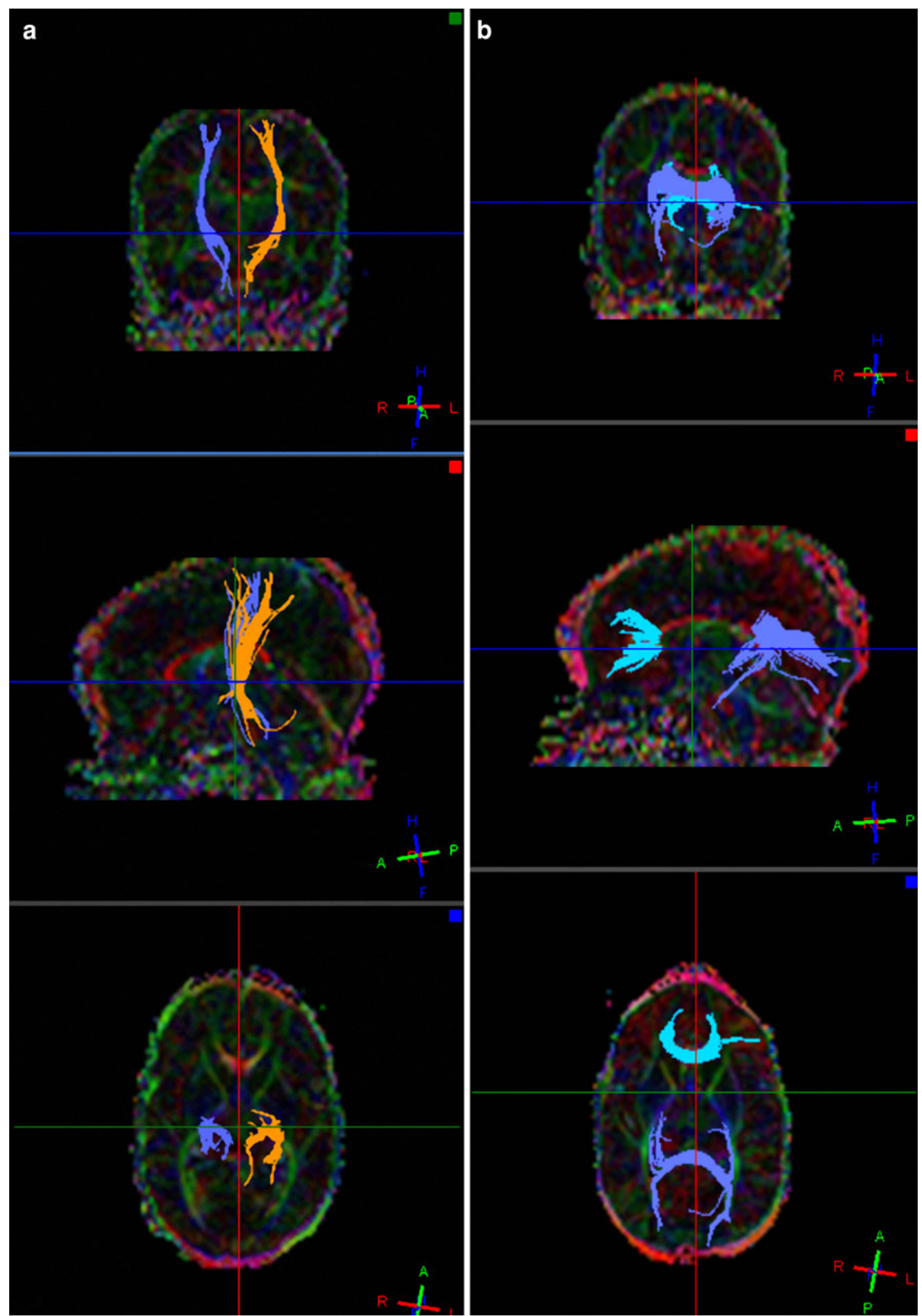

Fig. 2 a-b: Fibre tracts in an infant undergoing imaging at PMA of 41 weeks a through the PLIC and $\mathbf{b}$ through the genu and splenium of the $\mathrm{CC}$ using an automated 3D seeded technique according to default settings (FiberTrak, Philips Medical Systems, Best the Netherlands; minimum FA 0.15, maximum angle change 27.0 degrees, minimum fibre length $10.0 \mathrm{~mm}$ ) 
Table 2 Average FA, ADC values and lengths of fibres through the PLIC (posterior limb internal capsule) and genu and splenium of the CC (corpus callosum) $(n=84)$

\begin{tabular}{llc}
\hline & Mean (SD) & Range \\
\hline PLIC & & \\
FA & $0.37(0.02)$ & $0.34-0.43$ \\
ADC $\left(10^{-3} \mathrm{~mm}^{2} / \mathrm{s}\right)$ & $1.06(0.05)$ & $0.95-1.23$ \\
Length $(\mathrm{mm})$ & $59.8(8.2)$ & $38.5-80.6$ \\
CC genu & & \\
FA & $0.37(0.04) * * *$ & $0.31-0.55$ \\
ADC $\left(10^{-3} \mathrm{~mm}^{2} / \mathrm{s}\right)$ & $1.32(0.10) * * *$ & $1.10-1.56$ \\
Length $\left(\mathrm{mm}^{2}\right)$ & $46.7(10.1) * * *$ & $26.8-79.6$ \\
CC splenium & & \\
FA & $0.40(0.04)$ & $0.34-0.51$ \\
ADC $\left(10^{-3} \mathrm{~mm}^{2} / \mathrm{s}\right)$ & $1.36(0.11)$ & $1.10-1.69$ \\
Length $(\mathrm{mm})$ & $59.3(13.1)$ & $27.5-80.2$ \\
\hline
\end{tabular}

$F A$ fractional anisotropy, $A D C$ apparent diffusion coefficient $* * *$ : significant difference $(p<0.001)$ between genu and splenium of the $\mathrm{CC}$

In a previous study by Dudink et al. of very low birth weight infants undergoing imaging within 4 days of birth an association was found between FA of the PLIC and GA. They found no association between ADC values and GA [16]. Although we did not find an association between FA or ADC of the PLIC and GA, we found a positive association between FA and PMA at MRI and a negative association between ADC and PMA at MRI. Increase in FA and decrease in ADC have been described before and reflect the (pre-)myelination and parallel organisation of fibres, such as expected in the development of the PLIC $[17,23,24]$. As we found no association with GA, the maturation of the PLIC seems to progress with age, independent of the degree of prematurity. Similar to our findings in the PLIC, no associations were found between DTI parameters of fibres passing through the CC and GA, while DTI parameters of the $\mathrm{CC}$ showed a significant association with PMA at MRI, except for the FA values of the genu. This indicates ongoing maturation of the splenium of the CC around TEA, again independent of GA, while for the genu this seems less clear. These findings are in agreement with the results of Partridge et al. who found no significant maturational trends of the genu of the $\mathrm{CC}$ in their serial study of 14 premature neonates with a GA between 25 and 34 weeks, of whom eight underwent a second MRI around TEA [24].

We found no associations between DTI values for the PLIC or CC and the degree of WM injury. These findings are only partly in agreement with the results of Hüppi et al., who at TEA found no difference in ADC values of WM between preterm infants with and without WM lesions. They found a $20 \%$ lower regional anisotropy in fibres descending from the internal capsule in children with WM injury compared with those without [18]. Our data do not confirm this latter finding. This may be explained by the fact that we studied a prospective cohort of unselected VPTI, with relatively small numbers of children with normal or severely abnormal WM. Hüppi et al. selected ten infants with WM injury and matched these with ten infants of the same GA and neonatal course but with normal MRI. Our results also differ from those of Counsell et al., who demonstrated in preterm infants who underwent imaging around TEA (38.86-43.86 weeks), that radial diffusivity in the PLIC and the splenium of the $\mathrm{CC}$ and axial and radial diffusivity in the WM were significantly elevated in infants with DEHSI compared with those with normal appearing WM and term control
Table 3 Spearman Rank correlation coefficients between DTI parameters for PLIC and CC and age, weight and head circumference at birth and MRI

\footnotetext{
$H C$ head circumference, $G A$ gestational age, $P M A$ postmenstrual age,

* significant association: $p<0.05, * *$ significant association: $p<0.01, * * *$ significant association: $p<0.001$
}

\begin{tabular}{lcccccc}
\hline & GA & Birth weight & HC at birth & PMA at MRI & Weight at MRI & HC at MRI \\
\hline PLIC & & & & & & \\
FA & 0.02 & -0.07 & -0.02 & $0.48 * * *$ & $0.30 * *$ & $0.29 * *$ \\
ADC & 0.06 & 0.12 & 0.05 & $-0.65 * * *$ & $-0.48 * * *$ & $-0.42 * * *$ \\
Length & -0.13 & -0.09 & -0.03 & $0.42 * * *$ & $0.30 * *$ & $0.34 * *$ \\
CC genu & & & & & & \\
FA & -0.03 & -0.15 & $-0.35 * *$ & 0.17 & 0.07 & 0.10 \\
ADC & 0.13 & $0.23 *$ & 0.20 & $-0.53 * * *$ & $-0.22 *$ & -0.14 \\
Length & -0.14 & -0.10 & -0.04 & $0.46 * * *$ & $0.35 * * *$ & $0.34 * *$ \\
CC splenium & & & & & \\
FA & -0.001 & -0.08 & -0.16 & $0.24 * *$ & 0.17 & 0.10 \\
ADC & -0.06 & 0.05 & -0.02 & $-0.35 * * *$ & -0.19 & -0.21 \\
Length & 0.09 & 0.05 & $0.33 *$ & $0.43 * * *$ & $0.42 * * *$ & $0.52 * * *$ \\
\hline
\end{tabular}



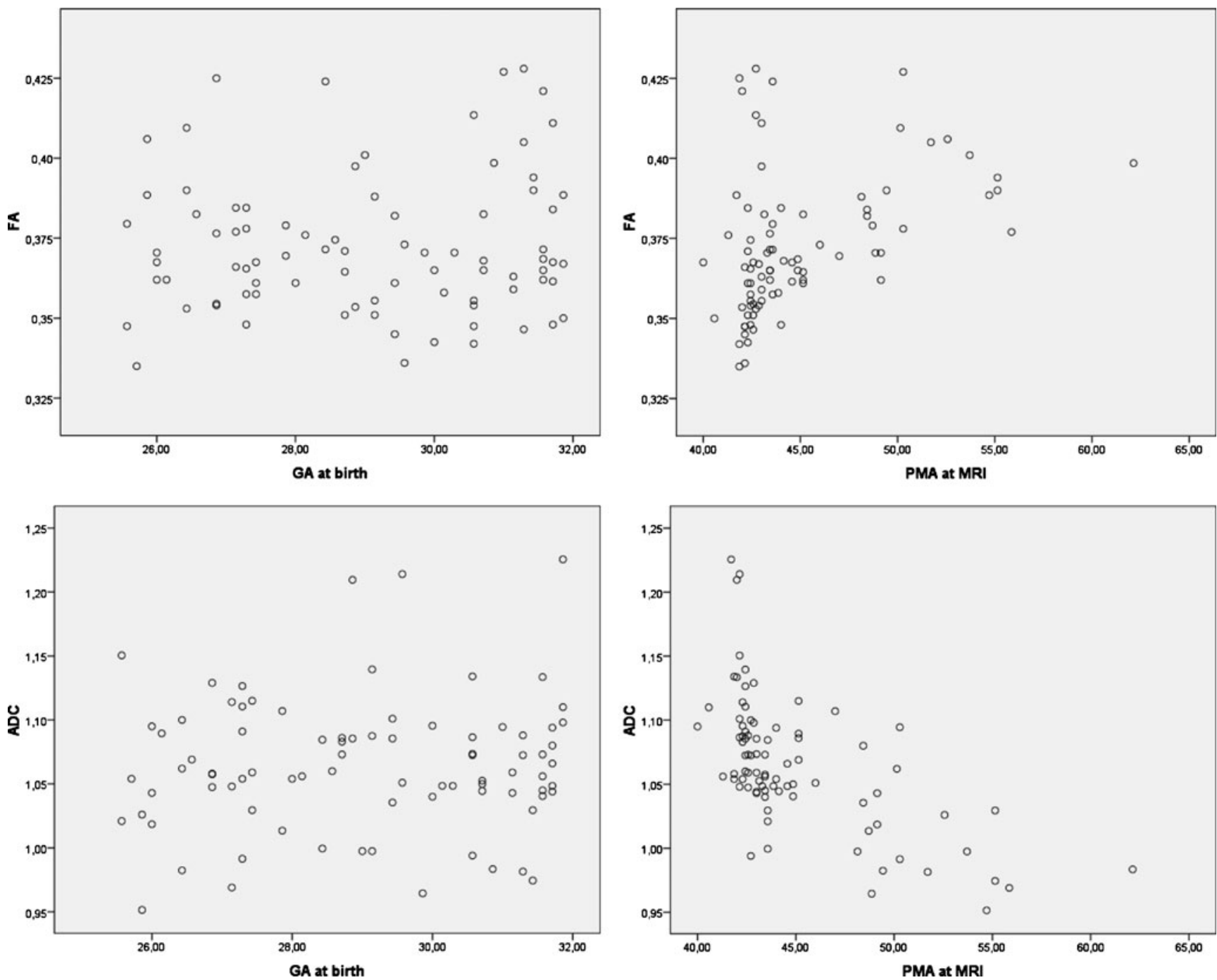

Fig. 3 Scatter plots of the individual data of the association between the FA and ADC of fibres through the PLIC and GA and PMA at MRI, showing no association with GA, a positive association between FA and PMA and a negative association between ADC and PMA

infants [37]. Counsell et al. randomly recruited 38 preterm infants and compared these with 8 healthy term born controls. The preterm infants were divided into a group with normal appearing WM and a group with DEHSI. Infants with overt WM injury were excluded from their study. The difference with our results may again be explained by the fact that we studied a group of unselected VPTI. It may also be hypothesised that the wide age range at imaging in our study group plays a role. However, as the results in the subgroup of infants undergoing imaging before 46 weeks were comparable to those in the whole group, we feel this will not have influenced our results. Possibly the unequal distribution of infants with normal/ mildly, moderately or severely abnormal WM has played a more important role. However, when comparing the 18 subjects with normal/mildly abnormal WM with the 15 with severe WM injury we found no difference between these two groups. In a recent study Cheong et al. [39] found a difference in FA in the PLIC in VPTI with extensive WM injury compared with VPTI with normal or focal WM injury using DTI region of interest measurements. In their study 39 infants had no WM signal intensity abnormalities, 59 infants had focal and 13 infants extensive WM abnormalities. The categorisation of WM injury we used is very similar to that of Cheong et al., but they included a large number of infants with normal appearing WM whereas that group was relatively small in our study.

Furthermore, in accordance with the study of Counsell et al [37] we did not identify any categorical clinical factors associated with DTI parameters. 
Table 4 Stepwise linear regression models for DTI parameters (PLIC and $\mathrm{CC}$ ), including the strongest contributing factors

\begin{tabular}{llllc}
\hline & $\mathrm{R}$ & $\mathrm{p}$ & Predictors & $\beta$ \\
\hline PLIC & & & & \\
FA & 0.51 & $<0.001$ & PMA at MRI & 0.002 \\
ADC & 0.63 & $<0.001$ & PMA at MRI & -0.008 \\
Length & 0.31 & $<0.01$ & HC at MRI & 1.27 \\
CC genu & & & & \\
FA & 0.37 & 0.02 & HC at birth & -0.008 \\
ADC & 0.55 & $<0.001$ & PMA at MRI & -0.013 \\
Length & 0.33 & $<0.01$ & PMA at MRI & 0.80 \\
CC splenium & & & & \\
FA & 0.23 & 0.04 & PMA at MRI & 0.002 \\
ADC & 0.41 & $<0.001$ & PMA at MRI & -0.01 \\
Length & 0.69 & $<0.001$ & PMA at MRI & 1.52 \\
& & & HC at birth & 3.02 \\
\hline
\end{tabular}

$P M A$ postmenstrual age, $H C$ head circumference

For the CC we found significantly different DTI values between the genu and splenium. In the genu FA and ADC values were significantly lower, indicating that at this young age the genu is less developed than the splenium. In terms of development similar findings have been reported by Barkovich, who pointed out that the myelination of the splenium precedes that of the genu on conventional MRI [9].

One of the main limitations of our study is that, due to clinical circumstances not all VPTI were imaged around
TEA. Therefore, although most were imaged before 46 weeks PMA (69/84), a wide age range existed. On the other hand, this enabled us to study the relation between PMA and DTI values. Another limitation of the present study is that we did not perform DTI in normal term infants. Therefore we were unable to compare the DTI values of the VPTI with those of a healthy control group.

We performed fibre tractography with commercially available software and with given default settings. Still, one of the limitations of fibre tracking is that in other studies different parameters or different tracking programs may be used, complicating the direct comparison of fibre tracking values between studies. Finally, as we performed fibre tractography of fibres passing through PLIC and CC, we only calculated DTI values in the WM tracts passing through these structures. We may therefore have missed a possible association between DTI parameters and either normal or injured WM.

In conclusion, our data show in VPTI imaged around or within 5 months of TEA, a strong association between DTI parameters of the PLIC and CC and PMA at imaging, but not between these values and GA. No association was found between DTI parameters of the PLIC and CC and the degree of WM injury or categorical clinical factors.

Although it has been reported that WM injury may result in atrophy of the PLIC and CC later on in life [3-7], our results indicate that in our cohort of VPTI, at this young age, the development of the PLIC and CC is ongoing and independent of the degree of prematurity or diffuse WM injury.

Table 5 DTI parameters for PLIC and CC and categorised WM injury $(n=84)$

\begin{tabular}{|c|c|c|c|c|c|c|c|}
\hline & $\begin{array}{l}\text { Normal-mild } \\
(n=18) \\
\text { Mean(SD) }\end{array}$ & $\begin{array}{l}\text { Moderate } \\
(n=51)\end{array}$ & $\begin{array}{l}\text { Severe } \\
(n=15)\end{array}$ & $\begin{array}{l}\text { Overall } \\
\text { p value }\end{array}$ & $\begin{array}{l}\text { Normal-Mild vs. } \\
\text { Severe }\end{array}$ & $\begin{array}{l}\text { Normal-Mild vs. } \\
\text { Moderate }\end{array}$ & $\begin{array}{l}\text { Moderate vs. } \\
\text { Severe }\end{array}$ \\
\hline \multicolumn{8}{|l|}{ PLIC } \\
\hline FA & $0.38(0.02)$ & $0.37(0.02)$ & $0.37(0.03)$ & 0.27 & 1.00 & 0.34 & 1.00 \\
\hline $\mathrm{ADC}$ & $1.05(0.05)$ & $1.07(0.05)$ & $1.07(0.04)$ & 0.64 & 1.00 & 0.73 & 1.00 \\
\hline Length & $59.4(8.0)$ & $60.0(7.0)$ & $59.6(11.9)$ & 0.96 & 1.00 & 0.76 & 1.00 \\
\hline \multicolumn{8}{|c|}{ CC genu } \\
\hline FA & $0.38(0.05)$ & $0.37(0.04)$ & $0.38(0.04)$ & 0.58 & 1.00 & 1.00 & 1.00 \\
\hline $\mathrm{ADC}$ & $1.30(0.11)$ & $1.32(0.09)$ & $1.33(0.10)$ & 0.65 & 1.00 & 1.00 & 1.00 \\
\hline Length & $45.6(11.1)$ & $47.4(9.6)$ & $45.6(11.1)$ & 0.73 & 1.00 & 1.00 & 1.00 \\
\hline \multicolumn{8}{|c|}{ CC splenium } \\
\hline FA & $0.40(0.04)$ & $0.39(0.03)$ & $0.41(0.03)$ & 0.54 & 1.00 & 1.00 & 0.83 \\
\hline $\mathrm{ADC}$ & $1.32(0.09)$ & $1.36(0.12)$ & $1.39(0.11)$ & 0.16 & 0.17 & 0.60 & 0.84 \\
\hline Length & $62.7(12.2)$ & $59.3(12.7)$ & $55.2(15.2)$ & 0.27 & 0.31 & 1.00 & 0.84 \\
\hline
\end{tabular}

PLIC posterior limb of the internal capsule, $C C$ corpus callosum, $W M$ white matter.

WM injury was categorised: normal/mildly abnormal, moderately abnormal and severely abnormal. 
Acknowledgements We would like to thank Wouter Teeuwisse for his assistance in developing the DTI protocol and his help acquiring the DTI datasets.

Open Access This article is distributed under the terms of the Creative Commons Attribution Noncommercial License which permits any noncommercial use, distribution, and reproduction in any medium, provided the original author(s) and source are credited.

\section{References}

1. Khwaja O, Volpe JJ (2008) Pathogenesis of cerebral white matter injury of prematurity. Arch Dis Child Fetal Neonatal Ed 93:F153F161

2. Counsell SJ, Rutherford MA, Cowan FM, Edwards AD (2003) Magnetic resonance imaging of preterm brain injury. Arch Dis Child Fetal Neonatal Ed 88:F269-F274

3. Barkovich AJ, Kjos BO (1988) Normal postnatal development of the corpus callosum as demonstrated by MR imaging. AJNR Am J Neuroradiol 9:487-491

4. Nosarti C, Rushe TM, Woodruff PW, Stewart AL, Rifkin L, Murray RM (2004) Corpus callosum size and very preterm birth: relationship to neuropsychological outcome. Brain 127:2080-2089

5. Rademaker KJ, Lam JN, Van H, Uiterwaal CS, Lieftink AF, Groenendaal F, Grobbee DE, De Vries LS (2004) Larger corpus callosum size with better motor performance in prematurely born children. Semin Perinatol 28:279-287

6. Cowan FM, De Vries LS (2005) The internal capsule in neonatal imaging. Semin Fetal Neonatal Med 10:461-474

7. Gilmore JH, Lin W, Corouge I, Vetsa YS, Smith JK, Kang C, Gu H, Hamer RM, Lieberman JA, Gerig G (2007) Early postnatal development of corpus callosum and corticospinal white matter assessed with quantitative tractography. AJNR Am J Neuroradiol 28:1789-1795

8. Barkovich AJ (2005) Magnetic resonance techniques in the assessment of myelin and myelination. $\mathrm{J}$ Inherit Metab Dis 28:311-343

9. Barkovich AJ, Maroldo TV (1993) Magnetic resonance imaging of normal and abnormal brain development. Top Magn Reson Imaging 5:96-122

10. Barkovich AJ, Kjos BO, Jackson DE Jr, Norman D (1988) Normal maturation of the neonatal and infant brain: MR imaging at $1.5 \mathrm{~T}$. Radiology 166:173-180

11. Sie LT, van der Knaap MS, van Wezel-Meijler G, Valk J (1997) MRI assessment of myelination of motor and sensory pathways in the brain of preterm and term-born infants. Neuropediatrics 28:97-105

12. Battin MR, Maalouf EF, Counsell SJ, Herlihy AH, Rutherford MA, Azzopardi D, Edwards AD (1998) Magnetic resonance imaging of the brain in very preterm infants: visualization of the germinal matrix, early myelination, and cortical folding. Pediatrics 101:957-962

13. Counsell SJ, Maalouf EF, Fletcher AM, Duggan P, Battin M, Lewis HJ, Herlihy AH, Edwards AD, Bydder GM, Rutherford MA (2002) MR imaging assessment of myelination in the very preterm brain. AJNR Am J Neuroradiol 23:872-881

14. Rutherford M, Malamateniou C, Zeka J, Counsell S (2004) MR imaging of the neonatal brain at 3 Tesla. Eur J Paediatr Neurol 8:281-289

15. Rutherford MA, Ward P, Malamatentiou C (2005) Advanced MR techniques in the term-born neonate with perinatal brain injury. Semin Fetal Neonatal Med 10:445-460
16. Dudink J, Lequin M, van Pul C, Buijs J, Conneman N, van Goudoever J, Govaert P (2007) Fractional anisotropy in white matter tracts of very-low-birth-weight infants. Pediatr Radiol 37:1216-1223

17. Huppi PS, Dubois J (2006) Diffusion tensor imaging of brain development. Semin Fetal Neonatal Med 11:489-497

18. Huppi PS, Murphy B, Maier SE, Zientara GP, Inder TE, Barnes PD, Kikinis R, Jolesz FA, Volpe JJ (2001) Microstructural brain development after perinatal cerebral white matter injury assessed by diffusion tensor magnetic resonance imaging. Pediatrics 107:455-460

19. Huppi PS, Maier SE, Peled S, Zientara GP, Barnes PD, Jolesz FA, Volpe JJ (1998) Microstructural development of human newborn cerebral white matter assessed in vivo by diffusion tensor magnetic resonance imaging. Pediatr Res 44:584-590

20. Glenn OA, Ludeman NA, Berman JI, Wu YW, Lu Y, Bartha AI, Vigneron DB, Chung SW, Ferriero DM, Barkovich AJ, Henry RG (2007) Diffusion tensor MR imaging tractography of the pyramidal tracts correlates with clinical motor function in children with congenital hemiparesis. AJNR Am J Neuroradiol 28:1796-1802

21. Glenn OA, Henry RG, Berman JI, Chang PC, Miller SP, Vigneron DB, Barkovich AJ (2003) DTI-based three-dimensional tractography detects differences in the pyramidal tracts of infants and children with congenital hemiparesis. J Magn Reson Imaging 18:641-648

22. Watts R, Liston C, Niogi S, Ulug AM (2003) Fiber tracking using magnetic resonance diffusion tensor imaging and its applications to human brain development. Ment Retard Dev Disabil Res Rev 9:168-177

23. Berman JI, Mukherjee P, Partridge SC, Miller SP, Ferriero DM, Barkovich AJ, Vigneron DB, Henry RG (2005) Quantitative diffusion tensor MRI fiber tractography of sensorimotor white matter development in premature infants. Neuroimage 1-10-27:862-871

24. Partridge SC, Mukherjee P, Henry RG, Miller SP, Berman JI, Jin H, Lu Y, Glenn OA, Ferriero DM, Barkovich AJ, Vigneron DB (2004) Diffusion tensor imaging: serial quantitation of white matter tract maturity in premature newborns. Neuroimage 22:1302-1314

25. Partridge SC, Mukherjee P, Berman JI, Henry RG, Miller SP, Lu Y, Glenn OA, Ferriero DM, Barkovich AJ, Vigneron DB (2005) Tractography-based quantitation of diffusion tensor imaging parameters in white matter tracts of preterm newborns. J Magn Reson Imaging 22:467-474

26. Ben BD, Ben SL, Graif M, Pianka P, Hendler T, Cohen Y, Assaf Y (2005) Normal white matter development from infancy to adulthood: comparing diffusion tensor and high $\mathrm{b}$ value diffusion weighted MR images. J Magn Reson Imaging 21:503-511

27. Provenzale JM, Liang L, DeLong D, White LE (2007) Diffusion tensor imaging assessment of brain white matter maturation during the first postnatal year. AJR Am J Roentgenol 189:476-486

28. Schneider JF, Il'yasov KA, Hennig J, Martin E (2004) Fast quantitative diffusion-tensor imaging of cerebral white matter from the neonatal period to adolescence. Neuroradiology 46:258-266

29. Rollins NK (2007) Clinical applications of diffusion tensor imaging and tractography in children. Pediatr Radiol 37:769-780

30. Rutherford M, Srinivasan L, Dyet L, Ward P, Allsop J, Counsell S, Cowan F (2006) Magnetic resonance imaging in perinatal brain injury: clinical presentation, lesions and outcome. Pediatr Radiol 36:582-592

31. Rutherford MA, Cowan FM, Manzur AY, Dubowitz LM, Pennock JM, Hajnal JV, Young IR, Bydder GM (1991) MR imaging of anisotropically restricted diffusion in the brain of neonates and infants. J Comput Assist Tomogr 15:188-198

32. Rutherford MA, Pennock JM, Counsell SJ, Mercuri E, Cowan FM, Dubowitz LM, Edwards AD (1998) Abnormal magnetic resonance signal in the internal capsule predicts poor neurodevelopmental outcome in infants with hypoxic-ischemic encephalopathy. Pediatrics 102:323-328 
33. Arzoumanian Y, Mirmiran M, Barnes PD, Woolley K, Ariagno RL, Moseley ME, Fleisher BE, Atlas SW (2003) Diffusion tensor brain imaging findings at term-equivalent age may predict neurologic abnormalities in low birth weight preterm infants. AJNR Am J Neuroradiol 24:1646-1653

34. Anjari M, Srinivasan L, Allsop JM, Hajnal JV, Rutherford MA, Edwards AD, Counsell SJ (2007) Diffusion tensor imaging with tract-based spatial statistics reveals local white matter abnormalities in preterm infants. Neuroimage 15-4-35:1021-1027

35. Bassi L, Ricci D, Volzone A, Allsop JM, Srinivasan L, Pai A, Ribes C, Ramenghi LA, Mercuri E, Mosca F, Edwards AD, Cowan FM, Rutherford MA, Counsell SJ (2008) Probabilistic diffusion tractography of the optic radiations and visual function in preterm infants at term equivalent age. Brain 131:573-582

36. Berman JI, Glass HC, Miller SP, Mukherjee P, Ferriero DM, Barkovich AJ, Vigneron DB, Henry RG (2009) Quantitative Fiber Tracking Analysis of the Optic Radiation Correlated with Visual Performance in Premature Newborns. AJNR Am J Neuroradiol 30:120-124

37. Counsell SJ, Shen Y, Boardman JP, Larkman DJ, Kapellou O, Ward P, Allsop JM, Cowan FM, Hajnal JV, Edwards AD, Rutherford MA (2006) Axial and radial diffusivity in preterm infants who have diffuse white matter changes on magnetic resonance imaging at term-equivalent age. Pediatrics 117:376386

38. Counsell SJ, Dyet LE, Larkman DJ, Nunes RG, Boardman JP, Allsop JM, Fitzpatrick J, Srinivasan L, Cowan FM, Hajnal JV, Rutherford MA, Edwards AD (2007) Thalamo-cortical connectivity in children born preterm mapped using probabilistic magnetic resonance tractography. Neuroimage 34:896904

39. Cheong JL, Thompson DK, Wang HX, Hunt RW, Anderson PJ, Inder TE, Doyle LW (2009) Abnormal white matter signal on MR imaging is related to abnormal tissue microstructure. AJNR Am J Neuroradiol 30:623-628

40. van Wezel-Meijler G, Leijser LM, de Bruine FT, Steggerda SJ, van der Grond J, Walther FJ (2009) Magnetic resonance imaging of the brain in newborn infants: practical aspects. Early Hum Dev 85:85-92

41. Leijser LM, de Bruine FT, van der Grond J, Steggerda SJ, Walther FJ, van Wezel-Meijler G (2010) Is sequential cranial ultrasound reliable for detection of white matter injury in very preterm infants? Neuroradiology 52:397-406 